\title{
A new description of lattice Yang-Mills theory and non-Abelian monopoles as the quark confiner
}

\author{
Akihiro Shibata* \\ Computing Research Center, High Energy Accelerator Research Organization (KEK) \& \\ Graduate University for Advanced Studies (Sokendai), Tsukuba 305-0801, Japan \\ E-mail: akihiro.shibata@kek.jp

\section{Kei-Ichi Kondo} \\ Department of Physics, Graduate School of Science, Chiba University, Chiba 263-8522, Japan \\ E-mail: kondok@faculty.chiba-u.jp
}

\section{Seikou Kato}

Takamatsu National College of Technology, Takamatsu 761-8058, Japan

E-mail: kato@takamatsu-nct.ac.jp

\section{Shoichi Ito}

Nagano National College of Technology, 716 Tokuma, Nagano 381-8550, Japan

E-mail: shoichidei.nagano-nct.ac.jp

\section{Toru Shinohara}

Graduate School of Science, Chiba University, Chiba 263-8522, Japan

E-mail: inohara@graduate.chiba-u.jp

\section{Takeharu Murakami}

Graduate School of Science, Chiba University, Chiba 263-8522, Japan

E-mail: tomefullmoon.sakura.ne.jp

\begin{abstract}
We propose a new description of the $S U(N)$ Yang-Mills theory on a lattice, which enables one to explain quark confinement based on the dual superconductivity picture in a gauge independent way. This is because we can define gauge-invariant magnetic monopoles which are inherent in the Wilson loop operator. For $S U(3)$ there are two options: the minimal option with a single type of non-Abelian magnetic monopole characterized by the maximal stability subgroup $\tilde{H}=U(2)=S U(2) \times U(1)$, and the maximal one with two types of Abelian magnetic monopoles characterized by the maximal torus subgroup $\tilde{H}=U(1) \times U(1)$. The maximal option corresponds to a gauge independent reformulation of the Abelian projection represented by the conventional MAG. In the minimal option, we have successfully performed the numerical simulation of the $S U(3)$ Yang-Mills theory on a lattice. We give preliminary numerical results showing the dominance of the non-Abelian magnetic monopole in the string tension obtained from the Wilson loop in the fundamental representation, and the infrared dominance of a decomposed field variable for correlation functions after demonstrating the preservation of color symmetry which was explicitly broken by the conventional MAG.
\end{abstract}

keywords: dual superconductivity, quark confinement, monopole dominance

The XXVI International Symposium on Lattice Field Theory

July 14-19 2008

Williamsburg, Virginia, USA

\footnotetext{
*Speaker.
} 


\section{Introduction}

The dual superconductivity which is believed as the promising mechanism for quark confinement is conjectured to occur due to the condensation of magnetic monopoles, just as the ordinary superconductivity is caused by the condensation of the Cooper pairs[1][2]. In the dual superconductor, the dual Meissner effect squeezes the color electric flux between a quark and an antiquark into a tube like region to form the hadronic string. The relevant data supporting the validity of this picture have been accumulated by numerical simulations especially since 1990 and some of the theoretical predications have been confirmed by these investigations; the infrared Abelian dominance, magnetic monopole dominance, center vortex dominance and non-vanishing off-diagonal gluon mass, which are the most characteristic features for dual superconductivity[3][4][5]. However, they are confirmed only in specific gauges such as the maximal Abelian (MA), Maximal Center (MC) and Laplacian Abelian gauge, which break color symmetry.

We give a new description of the Yang-Mills fields theory on a lattice, which is expected to give an efficient framework to explain quark confinement based on the dual superconductivity picture. The description enables us to extract in a gauge-independent manner the dominant degrees of freedom that are relevant to quark confinement in the Wilson criterion in such a way that they reproduce almost all the string tension in the linear inter-quark potential. We have already given a new framework for the lattice $S U$ (2) Yang-Mills theory as a lattice version of the Cho-Faddeev-Niemi-Shabanov (CFNS) decomposition in a continuum theory, and presented numerical evidences for its validity by performing numerical simulations. [6][7]

The issue of generating magnetic monopoles in $S U(N)$ Yang-Mills theory has been investigated so far under the MAG which breaks the original gauge group $S U(N)$ into the maximal torus subgroup $H=U(1)^{N-1}$. Then, MAG yields $N-1$ types of Abelian magnetic monopole, in agreement with the observation due to the homotopy group: $\pi_{2}\left(S U(N) / U(1)^{N-1}\right)=\pi_{1}\left(U(1)^{N-1}\right)=\mathbb{Z}^{N-1}$. Therefore, it tends to assume that magnetic monopoles of $N-1$ types are necessary to cause the dual Meissner effect for realizing quark confinement. However, it is not yet confirmed whether or not $N-1$ types of magnetic monopole are necessary to achieve confinement in $S U(N)$ Yang-Mills theory. Rather, we have a conjecture that a single type of magnetic monopole is sufficient to achieve quark confinement even in $S U(N)$ Yang-Mills theory, once it is defined in a gauge-invariant way. In fact, this scenario was originally proposed in [10] based on a non-Abelian Stokes theorem for the Wilson loop operator[11]. For $G=S U(3)$ there are two options: the minimal option with the maximal stability subgroup $\tilde{H}=$ $U(2)=S U(2) \times U(1)$ is a new one (overlooked so far) on which we focus in this Talk, while the maximal one with $\tilde{H}=U(1) \times U(1)$ being equal to the maximal torus subgroup corresponds to a gauge-independent reformulation of the Abelian projection represented by the conventional MAG as reported in the lattice 2007 conference[9].

\section{New variables on a lattice}

First, we summarize the result of a new description of the lattice $S U(N)$ Yang-Mills theory given in Ref.[8] as an extension of $S U(2)$ case. We wish to construct a lattice formulation in which an ordinary link variable $U_{x, \mu} \in G=S U(N)$ is decomposed in a gauge-independent manner into two variables $X_{x, \mu}$ and $V_{x, \mu}$, i.e., $U_{x, \mu}=X_{x, \mu} V_{x, \mu}$, so that only the variable $V_{x, \mu}$ carries the dominant contribution for quark confinement in agreement with the dual superconductivity picture. For this purpose, new variables in 
the relevant description are supposed to be transformed by a group element $\Omega_{x} \in G$ as

$$
\begin{aligned}
U_{x, \mu} & \rightarrow U_{x, \mu}^{\prime}=\Omega_{x} U_{x, \mu} \Omega_{x+\mu}^{\dagger}, \\
V_{x, \mu} \rightarrow V_{x, \mu}^{\prime} & =\Omega_{x} V_{x, \mu} \Omega_{x+\mu}^{\dagger}, \\
X_{x, \mu} & =X_{x, \mu}^{\prime}=\Omega_{x} X_{x, \mu} \Omega_{x}^{\dagger},
\end{aligned}
$$

where $V_{x, \mu}$ is defined as a link variable and transforms just like the original Yang-Mills link variable $U_{x, \mu}$, while $X_{x, \mu}$ is defined like a site variable representing a matter field and transforms according to the adjoint representation. It is important to see that decomposed variables are required to be transformed by the full $S U(3)$ gauge group. In the conventional Abelian projection, on the contrary, the $V_{x, \mu}$ field is identified with an Abelian part and is supposed to be transformed as an Abelian field.

The relationship between lattice variables and gauge variables in continuum theory [10] are given by

$$
\begin{aligned}
U_{x, \mu} & =X_{x, \mu} V_{x, \mu}=\exp \left(-i g \int d x^{\mu} \mathbf{A}_{\mu}(x)\right) \\
V_{x, \mu} & =\exp \left(-i g \int d x^{\mu} \mathbf{V}_{\mu}(x)\right)=\exp \left(-i g \varepsilon \mathbf{V}_{\mu}(x+\varepsilon \mu / 2)\right), \\
X_{x, \mu} & =\exp \left(-i g \varepsilon \mathbf{X}_{\mu}(x)\right) .
\end{aligned}
$$

In order to obtain the new variables by the decomposition respecting the gauge transformation property given in the above, we consider the extended Yang-Mills theory, called the master Yang-Mills theory (see Figure 1), by introducing a single type of the color field:

$$
\mathbf{h}_{x}:=\Theta_{x} \operatorname{diag}(1 / \sqrt{3}, 1 / \sqrt{3},-2 / \sqrt{3}) \Theta_{x}^{\dagger} \in S U(3) / U(2)=G / \tilde{H},
$$

such that it transforms according to the adjoint representation under an independent gauge transformation $\Theta_{x} \in G=S U(3)$. The decomposition $U=V X$ is determined by solving the defining equation;

$$
\begin{aligned}
& D_{\mu}^{\varepsilon}[V] \mathbf{h}_{x}:=\frac{1}{\varepsilon}\left(V_{x, \mu} \mathbf{h}_{x+\mu}-\mathbf{h}_{x} V_{x, \mu}\right)=0, \\
& \operatorname{tr}\left(X_{x, \mu} \mathbf{h}_{x}\right)=0
\end{aligned}
$$

where eq.(2.4a) represents that $\mathbf{h}(x)$ is covariantly constant in the background $V_{x, \mu}$ and eq.(2.4b) means that $X_{x, \mu}$ has the vanishing $\tilde{H}$-commutative part. ${ }^{1}$ The solution of the defining equations are obtained uniquely for given $U_{x, \mu}$ and $\mathbf{h}_{x}$ by way of a newly defined variable $\tilde{V}_{x, \mu}$ which does not belong to $S U(3)$ :

$$
\tilde{V}_{x, \mu}:=U_{x, \mu}+\frac{2 \sqrt{3}}{5}\left(\mathbf{h}_{x} U_{x, \mu}+U_{x, \mu} \mathbf{h}_{x+\mu}\right)+\frac{24}{5} \mathbf{h}_{x} U_{x, \mu} \mathbf{h}_{x+\mu} .
$$

In fact, the $S U(3)$ variable $V_{x, \mu}$ is obtained using the polar decomposition, together with another $S U(3)$ variable $X_{x, \mu}$ as

$$
V_{x, \mu}=\underline{V_{x, \mu}}\left(\underline{\operatorname{det} \underline{V_{x, \mu}}}\right)^{-1 / 3}, \quad \underline{V_{x, \mu}}:=\left(\tilde{V}_{x, \mu} \tilde{V}_{x, \mu}^{\dagger}\right)^{-1 / 2} \tilde{V}_{x, \mu}, \quad X_{x, u}=U_{x, \mu} V_{x, \mu}^{\dagger} .
$$

In order to obtain the equipollent theory (written in terms of the new variables) with the original YangMills theory, we impose the reduction condition which plays the role of reducing the extended gauge

\footnotetext{
${ }^{1}$ It is shown [12] using a non-Abelian Stokes theorem that a set of defining equations is obtained as a necessary and sufficient condition for the Wilson loop operator to be dominated by the decomposed variable $V_{x, \mu}$ in the sense $W_{C}\left[U_{x, \mu}\right] \cong$ (const.) $W_{C}\left[V_{x, \mu}\right]$.
} 


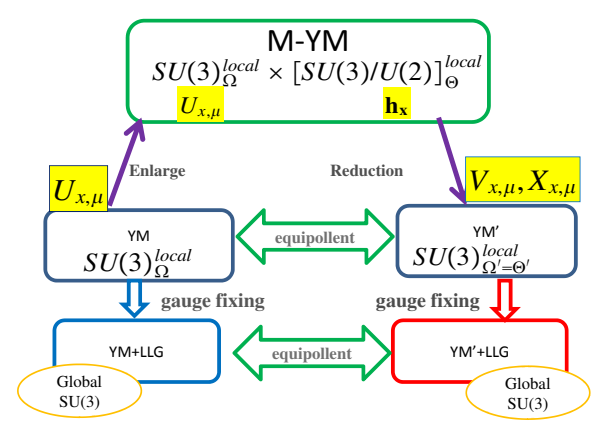

Figure 1: The master Yang-Mills theory:

symmetry $S U(3)_{\Omega} \times[S U(3) / U(2)]_{\Theta}$ to the original gauge symmetry $S U(3)_{\Omega=\Theta}$ so that the new variables $V_{x, \mu}, X_{x, \mu}$ and $\mathbf{h}_{x}$ transform under the same group $\Omega=\Theta \in S U(3)$. Such a reduction condition can be given by minimizing the functional $F_{R C}$ of $U_{x, \mu}$ and $\mathbf{h}_{x}$ under the independent local gauge transformations $\{\Omega, \Theta\}$ :

$$
F_{R C}\left[\Omega, \Theta ; U_{x, \mu}, \mathbf{h}_{x}\right]=\sum_{x, \mu} \operatorname{tr}\left(\left(D_{\mu}^{\varepsilon}\left[{ }^{\Omega} U_{x, \mu}\right]^{\Theta} \mathbf{h}_{x}\right)\left(D_{\mu}^{\varepsilon}\left[{ }^{\Omega} U_{x, \mu}\right]^{\Theta} \mathbf{h}_{x}\right)^{\dagger}\right) .
$$

By definition, the reduction condition (2.7) is invariant under the gauge transformation $\Theta=\Omega$ and does not break the original gauge symmetry as expected. Therefore, we can impose any gauge fixing afterwards.

\section{Numerical simulations}

Next, we proceed to the numerical simulations. The link variable configurations $\left\{U_{x, \mu}\right\}$ can be generated by using the standard method, since the decomposition $U_{x, \mu}=V_{x, \mu} X_{x, \mu}$ is done in a gaugeinvariant manner. The color field $\mathbf{h}_{x}$ is determined so as to fulfill the reduction condition using the same algorithm as that used in the the gauge fixing procedure. We have generated the link variable configurations for the Wilson lattice action using $16^{4}$ lattice at $\beta=5.70$, and obtained the new variables by imposing the lattice Landau gauge (LLG) for the original Yang-Mills field, rather than the MA gauge which breaks color symmetry explicitly.

We first focus on the color field. The new variables are defined so as to preserve the local gauge symmetry of the original Yang-Mills theory. Even after imposing LLG, therefore, the Yang-Mills theory should respect the global gauge symmetry, i.e., the color symmetry. This issue can be tested by measuring the vacuum expectation value (VEV) of the color field $\mathbf{h}_{x}$ and its correlation functions. The VEV of a color field shows $\left\langle h^{A}\right\rangle=0$ for $A=1,2, . ., N^{2}-1=8$. In Figure 2, we give a plot of correlation functions $\left\langle h^{A}(x) h^{B}(0)\right\rangle$ for $A, B=1, \ldots, 8$. All the diagonal parts $\left\langle h^{A}(x) h^{A}(0)\right\rangle$ have the same non-vanishing correlations, while all the off-diagonal parts $\left\langle h^{A}(x) h^{B}(0)\right\rangle(A \neq B)$ are vanishing. Thus, the correlation functions is of the form

$$
\left\langle h^{A}(x) h^{B}(0)\right\rangle=\delta^{A, B} D(x),
$$

which implies that the color symmetry is preserved. 

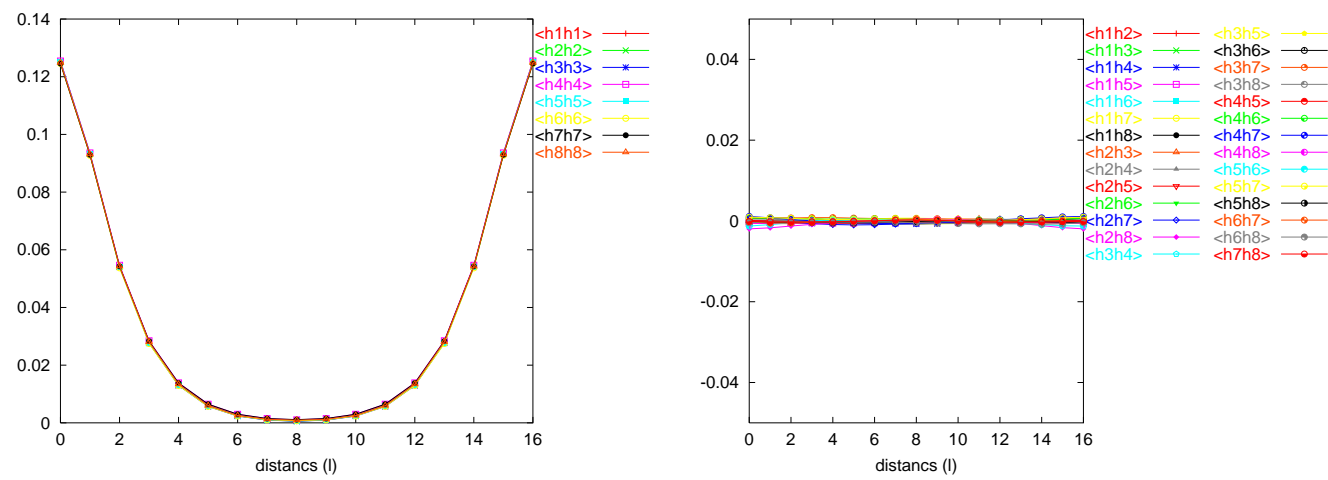

Figure 2: The correlation functions of the color field. (Left panel) diagonal parts, (Right panel) off-diagonal parts.

Next we investigate the static interquark potential through the new variables. The Wilson loop operator in the fundamental representation in the continuum theory is rewritten into [11]

$$
\begin{aligned}
W_{c}[\mathbf{A}] & =\operatorname{tr}\left[P \exp i g \oint_{C} \mathbf{A}_{\mu}(x) d x^{\mu}\right] / \operatorname{tr}(\mathbf{1})=\int d \mu[\xi]_{\Sigma} \exp \left\{i g \int_{\Sigma: \partial \Sigma=C} d S^{\mu v} F_{\mu v}[\mathbf{V}]\right\} \\
& =\int d \mu[\xi]_{\Sigma} \exp \left\{i g\left(K, \Xi_{\Sigma}\right)+i g\left(J, N_{\Sigma}\right)\right\}
\end{aligned}
$$

where we have defined $K:=\delta^{*} F, J:=\delta F, \Xi_{\Sigma}:=\delta^{*} \Theta_{\Sigma} \Delta^{-1}$ and $N_{\Sigma}:=\delta \Theta_{\Sigma} \Delta^{-1}$ with the fourdimensional Laplacian, $\Delta=d \delta+\delta d$. Here $\Theta_{\Sigma}$ is the vorticity tensor defined by $\Theta_{\Sigma}^{\mu v}=\int_{\Sigma} d S^{\mu v}(X(\sigma)) \delta(x-$ $X(\sigma))$ on the surface $\Sigma: \partial \Sigma=C$ spanned by the Wilson loop C. Note that $\mathbf{V}_{\mu}(x)$ field in eq(3.2) is equivalent to the $\mathbf{V}_{\mu}(x)$ reproduced from the new variable $V_{x, \mu}$ in the continuum limit. Therefore, the magnetic monopole contribution to the Wilson loop average on a lattice is given by

$$
\begin{aligned}
& \left\langle W_{C}[U]\right\rangle \cong\left\langle W_{C}[V]\right\rangle=\left\langle\exp \left(i \sum_{x, \mu} K_{x, \mu} \Xi_{x, \mu}\right)\right\rangle, \\
& \Xi_{x, \mu}=\sum_{s} \Delta^{-1}\left(s-s^{\prime}\right) \frac{1}{2} \varepsilon_{\mu \alpha \beta \gamma} \partial_{\alpha} S_{\beta \gamma}^{J}(s+\mu), \partial_{\alpha} S_{\alpha \beta}^{J}=J_{\beta},
\end{aligned}
$$

with the lattice magnetic monopole current $K_{x, \mu}$ given by

$$
K_{x, \mu}:=\frac{1}{2} \varepsilon_{\mu \lambda \alpha \beta} \partial_{\lambda} \Theta_{x, \alpha \beta}^{8}[V], \quad \Theta_{a \beta}^{8}[V]:=-\arg \operatorname{tr}\left(\left(\frac{1}{3} \mathbf{1}-\frac{2}{\sqrt{3}} h_{x}\right) V_{x, \alpha} V_{x+\alpha, \beta} V_{x+\beta, \alpha}^{\dagger} V_{x, \beta}^{\dagger}\right),
$$

where $\partial_{\lambda}$ denotes the forward difference (lattice derivative) in the $\lambda$ direction: $\partial_{\lambda} f(x):=f(x+\varepsilon \hat{\lambda})-$ $f(x)$. It should be noticed that the magnetic monopole current $K_{x, \mu}$ is gauge invariant, as can be seen from the transformation law of the new variables. This monopole should be identified with a "non-Abelian" magnetic monopole, since the monopole current is defined from $V_{x, \mu}$ which involves $\mathbf{h}_{x} \in G / \tilde{H}=S U(3) / U(2)$. The left panel of Figure 3 shows the distribution of the magnetic charge of the relevant magnetic monopoles obtained from 200 configurations, which have integral quantized values: $n_{x, \mu}=K_{x, \mu} / 2 \pi \in \mathbb{Z}$. The right panel of Figure 3 show the static potential calculated from the monopole part according to eq(3.3a). The numerical data of the static potential $V_{m}(R)$ extracted from $\left\langle\exp \left(i \sum_{x, \mu} K_{x, \mu} \Xi_{x, \mu}\right)\right\rangle$ is well fitted by a function $V_{m}(R)=-\alpha_{m} / R+\sigma_{m} R$ with the value $\sigma_{m}=$ $0.13301(36)$. In comparison with the full string tension, for example, the result in Ref.[13], $\sigma_{\text {full }}=$ 

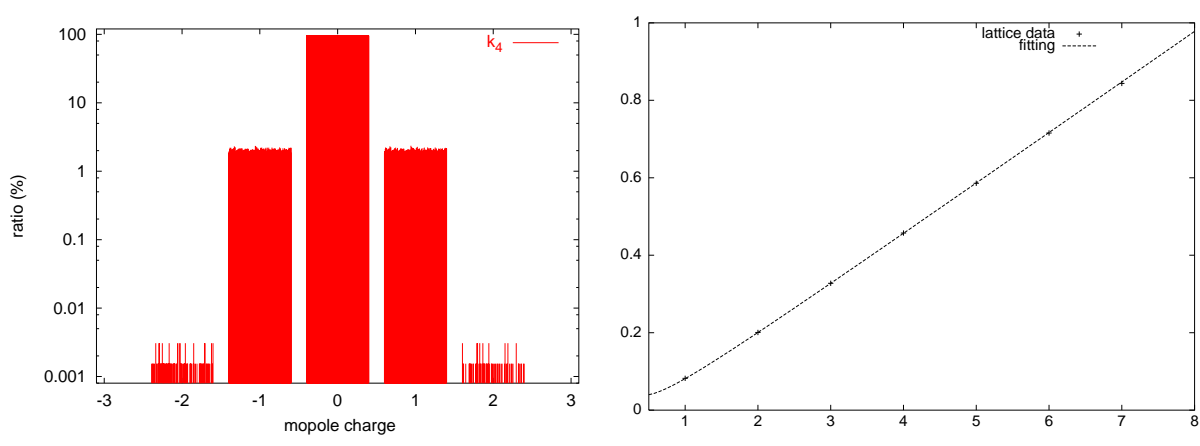

Figure 3: (Left panel) the magnetic monopole charge distribution, (Right panel) the static interquark potential calculated only from the magnetic monopole part.

0.3879(39), thus, we have shown the non-Abelian magnetic monopole dominance for the string tension in the $S U(3)$ Yang-Mills theory:

$$
\sigma_{m} / \sigma_{\text {full }}=0.87 \pm 0.19
$$

Finally, we devote to the correlation functions. The 2-point correlation functions (propagators) of the new variables and the original Yang-Mills gauge potential are defined by

$$
D_{O O}(x-y):=\left\langle O_{\mu}^{A}(x) O_{\mu}^{A}(y)\right\rangle \text { for } O_{\mu}^{A}\left(y^{\prime}\right) \in\left\{\mathbf{V}_{x^{\prime}, \mu}, \mathbf{X}_{x^{\prime}, \mu}, \mathbf{A}_{x^{\prime}, \mu}\right\}
$$

where an operator $O_{\mu}^{A}(x)$ is defined by the linear type, e.g., $\mathbf{A}_{x^{\prime}, \mu}:=\left(U_{x, \mu}-U_{x, \mu}^{\dagger}\right) / 2 g \varepsilon$. The left panel of Figure 4 shows the behavior of correlation functions, $D_{A A}, D_{V V}$ and $D_{X X}$ vs. the distance $l=|x-y|$. The correlation function $D_{V V}$ damps slowly and has almost the same damping as $D_{A A}$, while the $D_{X X}$ damps quickly. This result suggests that the $V$ part of the gluon propagator is dominated in the infrared region and mass generation by $X$ part. As the variable $X_{x, \mu}$ transforms as an adjoint matter, (see eq.(2.1c)), the Yang-Mills theory can have a gauge-invariant mass term $\mathscr{L}_{M_{X}}=M_{X}^{2} \operatorname{tr}\left(\mathbf{X}_{\mu}^{2}\right)$. The Fourier transformation of the massive propagator behaves for large $M_{X} r$ as

$$
D_{X X}(r)=\int \frac{d^{4} k}{(2 \pi)^{4}} e^{i k(x-y)} \frac{3}{k^{2}+M_{x}^{2}} \simeq \frac{3 \sqrt{M_{X}}}{2(2 \pi)^{3 / 2}} \frac{e^{-M_{X} r}}{r^{3 / 2}}
$$

and hence the scaled propagator $r^{3 / 2} D_{X X}(r)$ should be proportional to $\exp \left(-M_{X} r\right)$. The right panel of Figure 4 shows the logarithmic plot of the scaled propagators in LLG. This suggests the mass generation of gluons, although there exists the mixing between $V$ and $X$ in LLG and $M_{X}$ cannot be identified with the mass of $X$ straightforwardly.

\section{Summary and discussions}

We have given the new description of the lattice Yang-Mills theory to give a gauge independent decomposition of the link variable, $U_{x, \mu}=X_{x, \mu} V_{x, \mu}$, which approves the the gauge independent formulation of the dual superconductivity picture. We have performed the numerical simulation in the minimal case of the $S U(3)$ lattice Yang-Mills theory, and have demonstrated the color symmetry restoration, the non-Abelian monopole dominance in a gauge invariant way and the infrared $V$ dominance in LLG other than MAG. We have also shown based on a non-Abelian Stokes theorem that the string tension for the fundamental quark is explained by the non-Abelian magnetic monopole defined from $V$ part in the minimal case. 

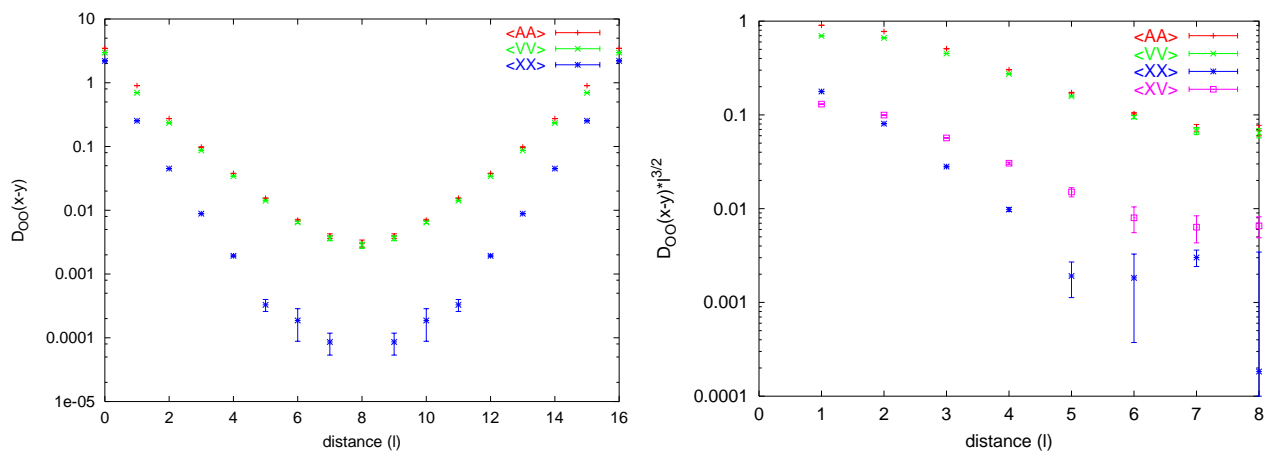

Figure 4: The correlation functions of original gauge field and new variables. (Left panel) The logplot of correlation functions. (Right panel) The scaled plot.

To establish the dual superconductivity picture by the new description of the lattice Yang-Mills theory, we need further study for the full $V$ dominance, e.g., "electric" and "magnetic" from the $V$ part reproduces the full string tension, the $\mathrm{N}$-ality property, and so on. These subjects are under investigation and will be discussed in a separate paper.

\section{Acknowledgement}

This work is financially supported by Grant-in-Aid for Scientific Research (C) 18540251 from Japan Society for the Promotion of Science (JSPS) and the Large Scale Simulation Program No. 08-16 (FY2008) of High Energy Accelerator Research Organization (KEK).

\section{References}

[1] Y. Nambu, Phys. Rev. D10, 4262 (1974); G. 't Hooft, in: High Energy Physics, edited by A. Zichichi (Editorice Compositori, Bologna, 1975); S. Mandelstam, Phys. Report 23, 245 (1976); A.M. Polyakov, (1975). Nucl. Phys. B120, 429 (1977).

[2] G. 't Hooft, Nucl.Phys. B190 [FS3], 455 (1981).

[3] Z.F. Ezawa and A. Iwazaki, Phys. Rev. D25, 2681 (1982).

[4] T. Suzuki and I. Yotsuyanagi, Phys. Rev. D42, 4257 (1990).

[5] J. Greensite, Eur. Phys. J. Spetial Topics 140 1-52 (2007)

[6] S. Ito, S. Kato, K.-I. Kondo, A. Shibata, T. Shinohara, Phys.Lett. B645 67-74 (2007)

[7] A. Shibata, S. Kato, K.-I. Kondo, T. Murakami, T. Shinohara, S. Ito, Phys.Lett. B653 101-108 (2007)

[8] Akihiro Shibata, Seiko Kato,Keiichi Kondo, Toru Shinohara and Shoichi Ito, Phys.Lett.B669:107-118 (2008),CHIBA-EP-168, KEK-2008-1,arXiv:0803.2451 [hep-lat]

[9] Akihiro Shibata, Seiko Kato,Keiichi Kondo, Toru Shinohara and Shoichi Ito, CHIBA-EP-166, KEK-PREPRINT-2007-50, POS(LATTICE-2007) 331, arXiv:0710.3221 [hep-lat]

[10] Kei-Ichi Kondo, Toru Shinohara, Takeharu Murakami, Prog.Theor.Phys.120 1-50 (2008), CHIBA-EP-167, arXiv:0803.0176 [hep-th]

[11] K.-I. Kondo, Phys.Rev.D77 085029 (2008) (arXiv:0801.1274 [hep-th])

[12] K.-I. Kondo and A. Shibata, CHIBA-EP-170, KEK-PREPRINT-2007-73, arXiv:0801.4203 [hep-th]

[13] R.G.Edward, U.M. Heller and T.R.Klassen, Nucl.Phys. N617 377-392 (1988) (arXir-hep-lat/9711003v2) 